\title{
1 Study of pre-synaptic internalisation in human schizophrenia \\ 2 brains
}

\section{Authors}

5 Makis Tzioras ${ }^{1,2}$, Anna J. Stevenson ${ }^{1,2}$, Delphine Boche ${ }^{3 *}$, Tara L. Spires-Jones ${ }^{1,2 *}$

\section{Affiliations}

$8 \quad{ }^{1}$ UK Dementia Research Institute, The University of Edinburgh, UK

$9{ }^{2}$ Centre for Brain Discovery Sciences, The University of Edinburgh, UK

$10{ }^{3}$ Clinical Neurosciences, Clinical and Experimental Sciences Academic Unit, Faculty of

11 Medicine, University of Southampton, UK

\section{* Equal contribution}

14 Correspondence to:

15 Professor Tara L. Spires-Jones

161 George Square, EH8 9JZ, Edinburgh, UK

17 Email: Tara.Spires-Jones@ed.ac.uk

18 Tel: 01316511895

\section{Abstract}

\section{Aims}

23 Efficient synaptic communication is crucial to maintain healthy behavioural and cognitive 24 processes. Individuals affected by schizophrenia present behavioural symptoms and 25 alterations in decision-making, suggesting altered synaptic integrity as the support of the 26 illness. It is currently unknown how this synaptic change is mediated in schizophrenia, but 27 microglia have been proposed to act as the culprit, actively removing synapses 28 pathologically. Here, we aimed to explore the interaction between microglia and synaptic uptake in human post-mortem tissue. 


\title{
34 Methods
}

35 We assessed microglial activation and synaptic internalisation by microglia in a post-mortem

36 human tissue of 10 control and 10 schizophrenia cases. Immunohistochemistry was

37 performed to identify microglia (Iba1 and CD68) and the presynaptic terminals (synapsin I).

\section{Results}

40 We found no difference in microglial expression, nor a difference in pre-synaptic protein

41 level phagocyted by microglia between the two groups.

\section{Conclusions}

44 Our findings are consistent with the brain imaging studies in schizophrenia implying that 45 microglia play a role mainly during the early phases of the disease, by example in active 46 synapse remodelling, which is not detected in the chronic stage of the illness.

\section{Keywords: synapse; gliosis; phagocytosis; psychiatric disorder; post-mortem;} immunohistochemistry

\author{
Abstract word count: 178 \\ Manuscript word count: 2,793 \\ Number of Figures: 2 \\ Number of Tables: 1
}




\section{Introduction}

71 Efficient synaptic communication is crucial to maintain healthy behavioural and cognitive

72 processes. In neurodevelopmental diseases, like schizophrenia, affected individuals can

73 exhibit behavioural symptoms like psychosis, hallucinations and alterations in decision-

74 making. A reduction in cortical grey matter volume and enlarged ventricles in the brains of

75 schizophrenia cases has been consistently reported [1,2]. This reduction in cortical volume is

76 likely to be an outcome of neuronal and synaptic loss, which has also been reported in

77 schizophrenia but the results have varied between brain area and synaptic markers examined

78 [3-7]. A meta-analysis of the expression of synaptic markers in the disease has shown

79 reduced levels of pre-synaptic markers, including synaptophysin and synapsin, in the

80 hippocampus and frontal cortex which are heavily implicated in schizophrenia, but not in

81 unaffected areas like the temporal and occipital lobes [8]. Synapses are crucial mediators of

82 brain communication [9-11], and so, such synaptic alterations can have an impact on brain

83 network connectivity, a process known to be affected in schizophrenia [12]. There are several

84 factors during brain development that influence brain connectivity, with non-neuronal

85 contributors playing an important role in synaptic formation and network maturation $[13,14]$.

86 One of these non-neuronal contributors are microglia, the resident brain immune cells and

87 primary phagocytes of the brain [14-17], which have been shown to facilitate neural network

88 shaping in development by phagocytosing synapses using the complement system [18-20].

89 However, microglia can be aberrantly involved in synaptic elimination in non-physiological

90 contexts, like observed in animal models of Alzheimer's disease [21,22]. In schizophrenia,

91 few experimental studies have explored the role of microglia in synaptic loss with evidence

92 to suggest their involvement in excessive synaptic pruning [23]. Whether this is true in the

93 human brain in schizophrenia is unknown. Here, we perform a human post-mortem study to

94 investigate the role of microglia in synaptic engulfment in schizophrenia. 


\section{Methods}

103

104

\section{Human tissue}

105 Ten cases with a confirmed diagnosis of schizophrenia and 10 non-neurological and non-

106 neuropathological controls were obtained from the Corsellis Collection (Table 1). Dorsal

107 prefrontal cortex, an area showing neuroimaging abnormalities with reduction of the grey

108 matter volume in chronic schizophrenia [1], was investigated for all cases. Cases with any

109 other significant brain pathologies such as infarct, tumour, or traumatic brain injury were

110 excluded from the study. Controls with no history of neurological or psychiatric disease or

111 symptoms of cognitive impairment were matched to cases as possible. No difference in age at

112 death and in post-mortem delay was detected between the 2 groups. To minimize the time in

113 formalin, which has an effect on the quality of the immunostaining, the selection was

114 performed on the availability of formalin-fixed paraffin embedded tissue, and thus on blocks

115 processed at the time of the original post-mortem examination. Characteristics of the cohorts

116 are provided in Table 1.

118 Table 1: Demographic, clinical and post-mortem characteristics of control and schizophrenia 119 cases

120

\begin{tabular}{l|c|c|c} 
Cases & $\mathbf{C t r l}(\mathbf{n}=\mathbf{1 0})$ & $\mathbf{S z}(\mathbf{n}=\mathbf{1 0})$ & P value \\
\hline Sex & $4 \mathrm{~F}: 6 \mathrm{M}$ & $2 \mathrm{~F}: 8 \mathrm{M}$ & \\
Age at death (years, mean $\pm \mathrm{SD})$ & $64.40 \pm 19.78$ & $64.80 \pm 20.37$ & $\mathrm{P}=0.94$ \\
Post-mortem delay (hours, mean $\pm \mathrm{SD})$ & $61.90 \pm 51.23$ & $50.60 \pm 24.52$ & $\mathrm{P}=0.61$ \\
Age of onset (years, mean $\pm \mathrm{SD})$ & $N A$ & $36.50 \pm 13.81$ & \\
Duration of illness & $N A$ & $35.13 \pm 21.85$ & \\
(years, mean $\pm \mathrm{SD})$ & & \\
Cause of death & 8 & 5 & \\
Cardiovascular disease & 1 & 3 & \\
infection/inflammation & 1 & 1 & \\
Trauma & 0 & & \\
Others $*$ & & & \\
\hline Ctrl, & & & \\
\hline
\end{tabular}

121 Ctrl, neurologically/cognitively normal controls; $S z$, Schizophrenia cases; $F$, female, $M$, male; $S D$, standard 122 deviation; NA, Not Applicable; *foreign body in respiratory tract 


\section{Immunohistochemistry}

125 Paraffin-embedded tissue was cut at $7 \mu \mathrm{m}$ thickness on a microtome and mounted on

126 Superfrost glass slides. The tissue was dewaxed in xylene, followed by rehydration in $100 \%$

$127 \mathrm{EtOH}, 90 \% \mathrm{EtOH}, 70 \% \mathrm{EtOH}, 50 \% \mathrm{EtOH}$, and finally water for 3 minutes each. Citric acid

128 pH6 (VectorLabs, H-3300) was used for heat-mediated antigen retrieval by pressure cooking

129 for 3 minutes at the steam setting. The slides were incubated for 5 minutes with

130 autofluorescence eliminator reagent (Merck Millipore, 2160) to reduce background, followed

131 by another 5 minutes incubation with Vector TrueView autofluorescence quenching kit

132 (VectorLabs, SP-8400) to reduce red blood cell autofluorescence. Sections were blocked in

$13310 \%$ normal donkey serum (Sigma Aldrich, D9663-10ML) and 0.3\% Triton X-100 (T8787-

134 100ML) for 1 hour at room temperature. Microglia were stained with Iba1 (Abcam, ab5079,

135 goat polyclonal, 1:500), and CD68 (DAKO, M0876, mouse monoclonal, 1:100), and pre-

136 synaptic terminals with synapsin I (Sigma Aldrich, AB1543P, rabbit polyclonal, 1:750),

137 overnight at $4^{\circ} \mathrm{C}$ in a humid chamber. All primary antibodies were diluted in the blocking

138 solution described above. The following cross-adsorbed secondary antibodies were used:

139 donkey anti-goat A647 (Thermo Fisher Scientific, A32849), donkey anti-mouse A594

140 (Thermo Fisher Scientific, A32744), and donkey anti-rabbit A488 (Thermo Fisher Scientific,

141 A32790). All secondary antibodies were diluted in phosphate buffer saline (PBS) (Thermo

142 Fisher Scientific, 70011036). For tissue washes, 10X PBS was diluted in water to $1 \mathrm{X}$

143 concentration, with the addition of $0.3 \%$ Triton X-100 in washes prior to primary antibody

144 incubation. Nuclei were counterstained with DAPI $(1 \mu \mathrm{g} / \mathrm{ml})$ (D9542-10MG, Sigma-Aldrich).

\section{Confocal microscopy and image analysis}

147 Twenty images were taken randomly throughout all cortical layers of the grey matter for each

148 case using a Leica TCS8 confocal microscope with a 63x oil immersion objective.

149 Acquisition parameters were kept constant for all images and cases. Lif files were split into

150 tiff files, and batch analysed on ImageJ (version 1.52p, Wayne Rasband, NIH, USA) using a

151 custom co-localisation and thresholding macro. Images from different cases were also

152 manually analysed to ensure the macro was accurately detecting positive signal and

153 excluding background. For synaptic internalisation by microglia we chose to analyse the

154 colocalization of CD68 with Syn1, and also normalised to either CD68 or Iba1 burden. Data

155 are expressed as protein burden (\%) defined as the area fraction of each image labelled by the 
156 antibody. 3D reconstruction images were generated in ParaView 5.8.0. All experiments and analyses were blinded to the experimenter.

Ethics

163 Ethical approval was provided by BRAIN UK, a virtual brain bank which encompasses the 164 archives of neuropathology departments in the UK and the Corsellis Collection, ethics 165 reference 14/SC/0098. The study was registered under the Ethics and Research Governance 166 (ERGO) of the Southampton University (Reference 19791).

\section{Statistics}

169 R Studio version 3.6.0 (2019-04-26) was used for statistical analysis [24]. Linear mixed170 effects models were used to examine the effect of disease status on microglial burdens and 171 CD68-Synapsin I co-localisation. This test was chosen because it allows all 20 images taken 172 per case to be considered while accounting for non-independence, instead of a single mean 173 value per case, allowing for a more powerful analysis on the results. QQ plots were generated 174 in R Studio to check the residuals were normally distributed, which is an assumption of the 175 mixed-effects model. To meet the assumptions of the test, all datasets were Tukey 176 transformed prior to analysis (untransformed data presented in graphs). GraphPad Prism 8 177 was used for generating bar graphs with a mean value plotted per case, represented as a dot. 178 We considered $\mathrm{p} \leq 0.05$ as significant. 


\section{Results}

\section{Microgliosis}

193 We studied post-mortem brains from 10 control (mean age $64.40 \pm 19.78$ ) and 10 confirmed

194 schizophrenia cases (mean age $64.80 \pm 20.37$ ) from dorsolateral pre-frontal cortex (DLPFC,

195 Brodmann area 46) which is affected in schizophrenia [1]. Gliosis is commonly observed

196 during loss of brain homeostasis. We examined microglial burden using Ibal which labels the

197 microglial cytoplasm and reflect microglial motility and homeostasis Iba1 is considered as a

198 pan-microglial marker and has been described increased in a subset of neurodegenerative

199 diseases [25]. The other microglial marker, CD68, labels the lysosomal compartment of

200 microglia revealing phagocytosis [26] (Figure 1A-B), and is increased in many neurological

201 diseases, including Alzheimer's disease [27] and stroke [28]. By thresholding for each of the

202 markers and quantifying their respective burdens, we found there was no difference in either

203 Iba1 ( $\mathrm{p}=0.315)$ or CD68 ( $\mathrm{p}=0.794)$ burdens between the schizophrenia and control cohorts

204 (Figure 1) (full statistical outcomes found in Supplementary Table 1). Furthermore, there was

205 no difference in the co-localisation between CD68 and Iba1 in controls and schizophrenia

206 brains $(\mathrm{p}=0.639)$, suggesting the co-expression of the two markers per single cell is

207 unchanged (Figure 1E). 



B


D

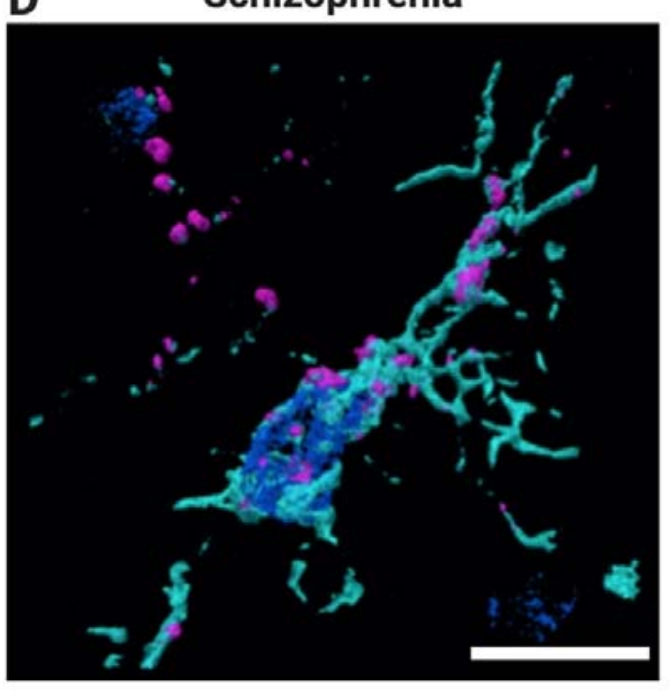

$\mathbf{E}$

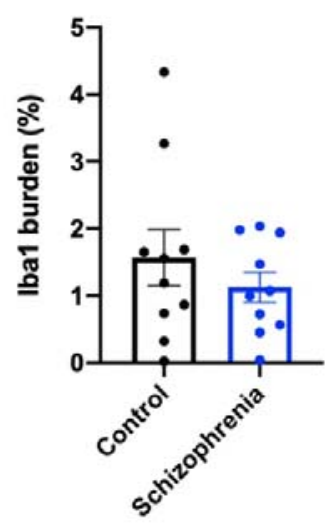

F

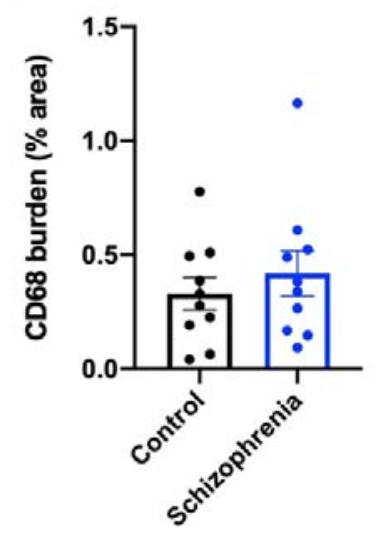

G

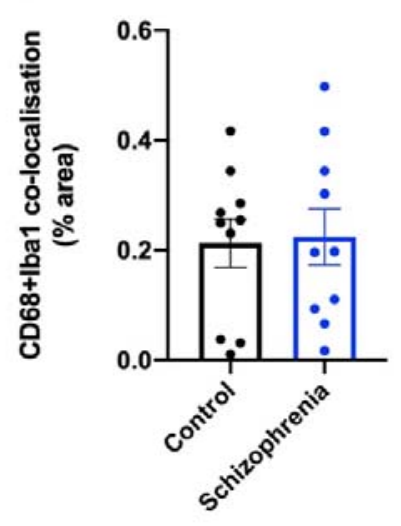


209 Figure 1. Microgliosis burdens unchanged in control and schizophrenia tissue.

210 Representative confocal images of immunohistochemistry stained sections for the microglial

211 markers Iba1 (cyan) and CD68 (magenta) in control (A and B) and schizophrenia (C and D)

212 tissue. Nuclei are counterstained with DAPI. Scale bars in large images represent $20 \mu \mathrm{m}$ and

$21310 \mu \mathrm{m}$ in the expanded inserts (denoted by dotted white lines). The insert images of A and B

214 are represented as 3D-reconstruction in B and D, respectively (scale bar, 10 $\mu \mathrm{m}$ ). 3-D

215 reconstructions made on ParaView. Quantification of Iba1 burdens (\% area), CD68 burdens

216 (\% area) and Iba1+CD68 co-expression (\% area) are shown in panels $\mathrm{E}$, F, and G,

217 respectively. Each data point represents a mean of 20 images taken per case, where $n=10$ per

218 group. Linear mixed-effects model showed no difference in microgliosis between the control

219 and schizophrenia cases in the above comparisons at a significance threshold of $\mathrm{p} \leq 0.05$.

\section{Synaptic engulfment}

225 Though no difference in microglial burdens between the two cohorts was observed, we aimed

226 to assess whether microglia were involved in synaptic engulfment in schizophrenia. To do

227 this, we quantified the amount of co-localisation between synapsin I and CD68 (\% area), as a

228 measure of engulfed synaptic material in the microglial phago-lysosomal compartment

229 (Figure 2). We found no difference in synaptic engulfment by microglia between the

230 schizophrenia and control cases $(\mathrm{p}=0.413)$ (Figure 2). Furthermore, when we normalised this

231 co-localisation to their respective CD68 or Iba1 burdens, there was still no statistical

232 difference between schizophrenia and control tissue ( $\mathrm{p}=0.167$ and $\mathrm{p}=0.964$ respectively)

233 (Figure 2). Our data therefore suggest that at the time of death, microglia do not appear to be

234 involved in aberrant synaptic internalisation in patients with schizophrenia. 

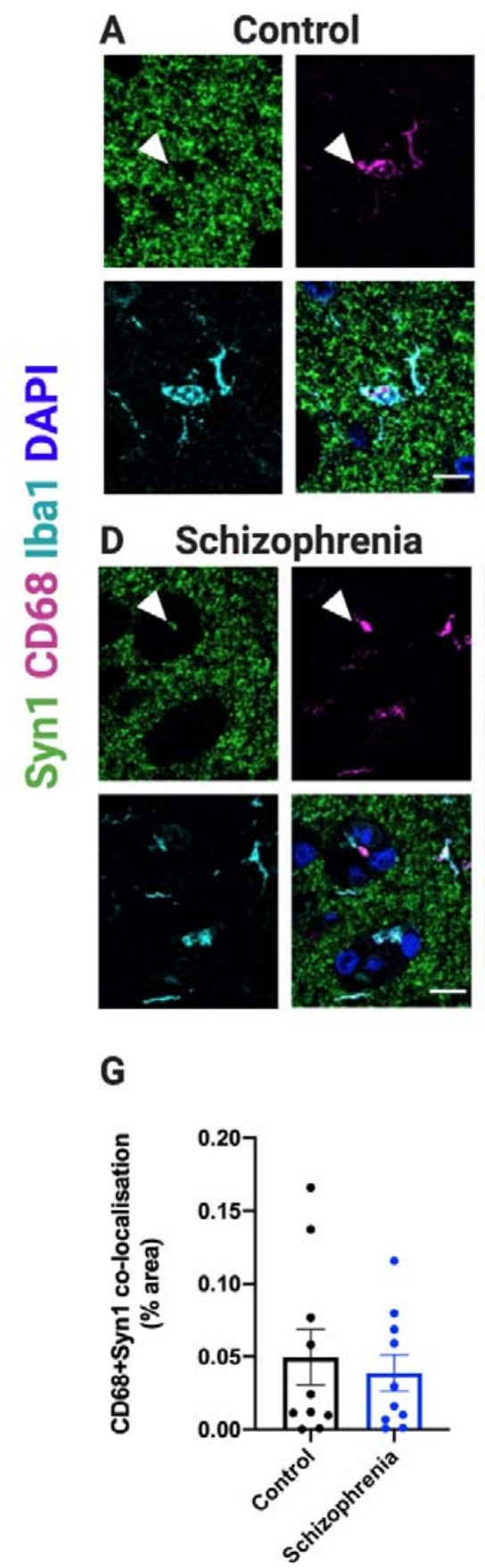

B



E



H

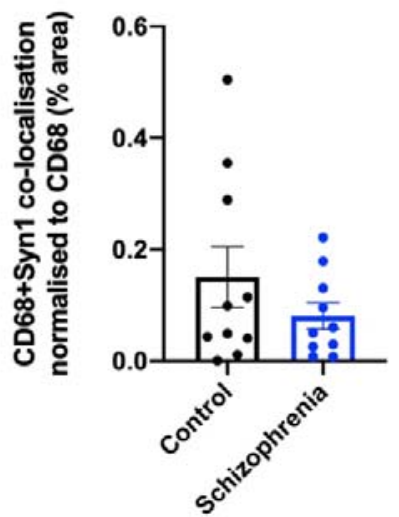

C

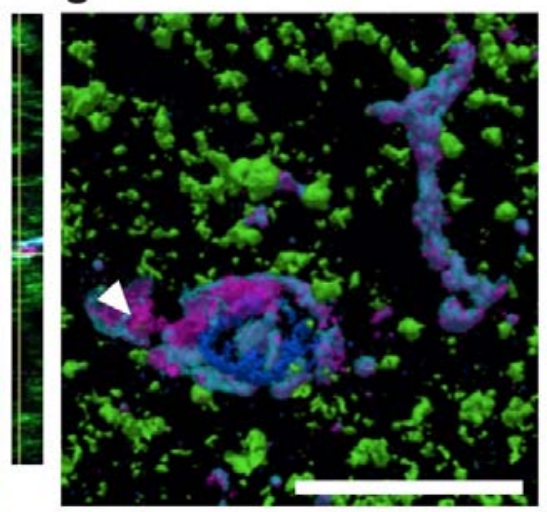

$\mathbf{F}$

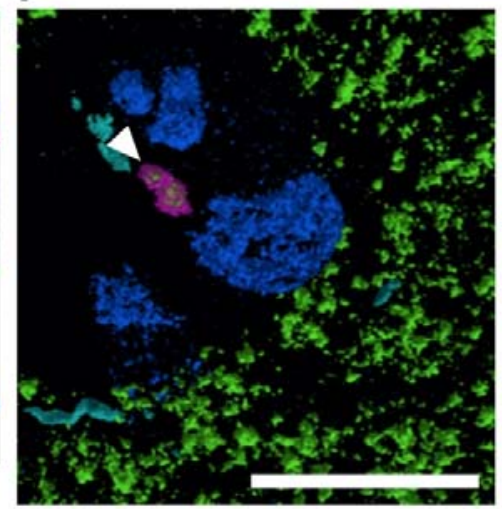

I

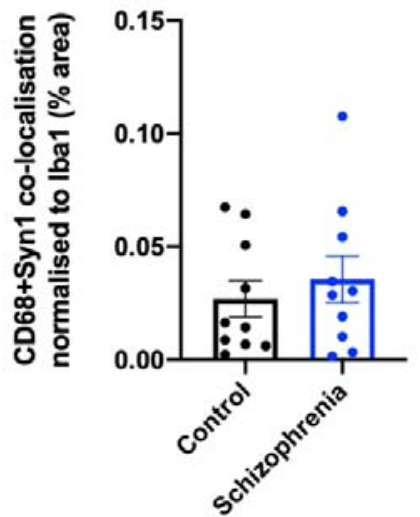

Figure 2. No difference found in synaptic engulfment by microglia in post-mortem tissue. Representative confocal images of the pre-synaptic marker synapsin I (green), CD68 (magenta) and Iba1 (cyan) in control (A-C) and schizophrenia tissue (D-F). Nuclei are counterstained with DAPI. A and D show individual panels of each stain and lastly the merged image, with white arrowheads pointing to sites of co-localisation between CD68 and synapsin I. B and E are expanded images of A and D, with orthogonal views indicating where CD68 and synapsin I co-localise. C and F represent 3D reconstructions from A and D, generated on ParaView. In G, the co-localisation index of CD68 and synapsin I is quantified 
are observed. By normalising each image to their respective CD68 burden or Iba1 burden there is still no statistical change in the engulfment of synapsin I by CD68. Each data point represents a mean average of 20 images taken per case, where $n=10$ per group. Linear mixedeffects model assessed statistical significance, considering $\mathrm{p} \leq 0.05$ for significance. All scale bars represent $10 \mu \mathrm{m}$.

\section{Discussion}

In human post-mortem tissue from both patients with schizophrenia and age-matched controls, we found pre-synaptic proteins inside microglial cells in the frontal cortex of the brain, but no difference in the levels of synaptic internalisation between the two groups.

A limitation of our post-mortem tissue is that it provides a snapshot of the disease, which lacks mechanistic insight. A greater sample size in an independent cohort will be useful to assess the reproducibility of these results and to stratify by confounding variables like sex and age. This would also allow assessment of comorbid symptoms in schizophrenia, like depression or psychosis, and if such symptoms affect microglial and synaptic internalisation by the cells. However, this study is unique, as schizophrenia tissue is scarce and by the type of assessment performed.

With gliosis being reported in multiple brain disorders, we assessed microgliosis in schizophrenia. As described above, we found no differences in microglial burdens between disease and control groups. This suggests that microglial activation is not a sustained event in chronic schizophrenia, and if any changes do occur in these cells it would instead likely involve functional alterations. Previous literature looking at CD68 expression in control and schizophrenia cases has also reported a similar outcome [29]. Given that schizophrenia is not a progressive, albeit chronic disease, it is understandable that if any changes in glial dynamics were to occur, they may be seen closer to disease onset, and that by the time the brains were donated 35 years later, any changes would have subsided. This would be consistent with the observations published to visualise and quantify microglial activation in vivo with positron emission computed tomography (PET) using specific ligands of the translocator protein TSPO [30]. The PET studies have revealed that activated microglia are present in patients within the first 5 years of disease onset or during a psychotic state, 
280 whereas other PET studies in chronic schizophrenia have shown no difference in microglial 281 activation between healthy controls and these patients.

282 Although developmental synaptic alterations, like synapse loss, have been characterised in 283 individuals with schizophrenia [3,8], there are key unanswered questions that remain. For 284 instance, it is not clear how the synapse elimination is mediated, the extent to which it drives 285 behavioural symptoms, or whether it is the outcome of other disease-specific pathologies. In 286 neurodegenerative diseases, such as Alzheimer's disease (AD), synapse loss is a hallmark of 287 the disease [31,32], and it has gained significant attention as it associates strongly with the 288 cognitive decline seen in patients [33,34]. Although schizophrenia and AD have very 289 different pathological features and the onset of the two disease is far apart, there are some common qualities that may help with understanding disease mechanisms. For example, a prominent mechanism for synaptic elimination in development is the use of the classical complement cascade (CCC), where it has been shown to sculpt neural circuits by tagging less electrically active synapses [18,20]. Recent research has now implicated complement as a signal for aberrant synapse elimination in disease [14,35]. Specifically, variants of C4 of the CCC are associated with a greater risk of developing schizophrenia [36], as well as poorer brain connectivity and schizophrenia-like behavioural deficits in mice [37].

Currently, a suggested mechanism by which complement-tagged synapses are cleared is by microglial recruitment for synaptic removal. The microglial importance in guiding cerebral circuitry has been recently described in a case study of a baby born with a homozygous mutation in the CSF1R gene causing a total lack of microglial cells, resulting in early death [38]. Upon autopsy, ectopic grey matter was found growing in the ventricles and impaired cell layer separation was observed in the grey matter, suggesting a faulty brain wiring due to loss of microglia. However, microglia can also be involved in abnormal synaptic elimination.

305 Indeed, increased microglial-mediated synaptic clearance has been observed in AD mouse 306 models [39] which was rescued in complement-deficient mice [21,40]. Of note, we have recently shown in human post-mortem tissue that in $\mathrm{AD}$ there is increased synaptic ingestion

308 by microglia, and that this is exacerbated in areas near amyloid- $\beta$ pathology [41]. In co309 cultured neuron and microglia-like cells from human induced pluripotent stem cells from 310 control and schizophrenia lines, increased levels of the excitatory post-synaptic protein PSD-

31195 was reported phagocytosed in the schizophrenia co-cultures [23]. Interestingly, this 312 increased phagocytic activity was mainly driven by the presence of schizophrenia-derived 313 microglia. Indeed, when schizophrenia neurons were co-cultured with microglia from control 
314 patients, the phagocytic index was reduced, indicating that in schizophrenia microglia have

315 intrinsic differences in their phagocytic response. It is worth noting that induced stem cells

316 are a good model for understanding human disease but represent a developmentally earlier

317 phenotype, and not that of the age of the donor. Therefore, this supports a role for phagocytic

318 microglia in early stages of the illness, and may explain why we did not see any changes in

319 phagocytic ability of microglia towards synapses in chronic schizophrenia, since we are not

320 studying the developmental time-frame.

322 In conclusion, here we report that microglia in human post-mortem tissue internalise pre323 synaptic proteins physiologically, and that this does not appear to be altered in the chronic 324 form of schizophrenia, at the difference to our observation in AD. Nevertheless, given the 325 typically early onset of schizophrenia and that synapse loss is likely to have occurred years 326 before brain collection, we cannot make assumptions on the role of microglia in synaptic 327 clearance at the start of the disease. Looking forward, it would be interesting to study 328 difference between young versus older cases in terms of synaptic uptake by microglia, and 329 phenotype these changes in several brain areas to investigate any region-specific differences. 330 Lastly, longitudinal PET imaging of the pre-synaptic marker SV2A [42-44] and TSPO 331 microglial marker would enable exploration of any microglia-synapse association during the 332 course of the illnesses. 


\section{Acknowledgements}

335 We would also like to thank our funders, specifically the UK Dementia Research Institute

336 which receives funding from Alzheimer's Research UK, the Alzheimer's Society, and the

337 Medical Research Council. We also would like to thank the Wellcome Trust for funding AJS

338 and TLSJ. Tissue samples were obtained from The Corsellis Collection as part of the UK

339 Brain Archive Information Network (BRAIN UK) which is funded by the Medical Research

340 Council and Brain Tumour Research.

341 Authors contributed in the following ways: MT contributed in study design, performed

342 experiments and imaging, statistical analysis, and manuscript preparation; AJS contributed in

343 statistical analysis and manuscript editing; DB contributed by providing cut paraffin-

344 embedded section, study design, and manuscript editing; TLSJ contributed with study design,

345 statistical analysis, and manuscript editing. Figures created with BioRender.

\section{References}

1 Kikinis Z, Fallon JH, Niznikiewicz M, Nestor P, Davidson C, Bobrow L, et al. Gray matter volume reduction in rostral middle frontal gyrus in patients with chronic schizophrenia. Schizophr Res 2010;123:153-9.

2 Kahn RS, Sommer IE, Murray RM, Meyer-Lindenberg A, Weinberger DR, Cannon TD, et al. Schizophrenia. Nat Rev Dis Primers 2015;1:15067.

3 Feinberg I. Schizophrenia: caused by a fault in programmed synaptic elimination during adolescence? J Psychiatr Res 1982;17:319-34.

4 Faludi G, Mirnics K. Synaptic changes in the brain of subjects with schizophrenia. Int J Dev Neurosci 2011;29:305-9.

5 Onwordi EC, Halff EF, Whitehurst T, Mansur A, Cotel M-C, Wells L, et al. Synaptic density marker SV2A is reduced in schizophrenia patients and unaffected by antipsychotics in rats. Nat Commun 2020;11:246.

6 Browning MD, Dudek EM, Rapier JL, Leonard S, Freedman R. Significant reductions in synapsin but not synaptophysin specific activity in the brains of some schizophrenics. Biol Psychiatry 1993;34:529-35.

7 Funk AJ, Mielnik CA, Koene R, Newburn E, Ramsey AJ, Lipska BK, et al. Postsynaptic Density-95 Isoform Abnormalities in Schizophrenia. Schizophr Bull 2017;43:891-9.

8 Osimo EF, Beck K, Reis Marques T, Howes OD. Synaptic loss in schizophrenia: a metaanalysis and systematic review of synaptic protein and mRNA measures. Mol Psychiatry 2019;24:549-61.

9 Wiesel TN, Hubel DH. Effects of visual deprivation on morphology and physiology of cells in the cats lateral geniculate body. J Neurophysiol 1963;26:978-93.

10 Engert F, Bonhoeffer T. Dendritic spine changes associated with hippocampal long-term synaptic plasticity. Nature 1999;399:66-70.

11 Matsuzaki M, Ellis-Davies GC, Nemoto T, Miyashita Y, lino M, Kasai H. Dendritic spine geometry is critical for AMPA receptor expression in hippocampal CA1 pyramidal 
neurons. Nat Neurosci 2001;4:1086-92.

12 Klauser P, Baker ST, Cropley VL, Bousman C, Fornito A, Cocchi L, et al. White matter disruptions in schizophrenia are spatially widespread and topologically converge on brain network hubs. Schizophr Bull 2017;43:425-35.

13 Eroglu C, Barres BA. Regulation of synaptic connectivity by glia. Nature 2010;468:22331.

14 Henstridge CM, Tzioras M, Paolicelli RC. Glial contribution to excitatory and inhibitory synapse loss in neurodegeneration. Front Cell Neurosci 2019;13:63.

15 Prinz M, Priller J. Microglia and brain macrophages in the molecular age: from origin to neuropsychiatric disease. Nat Rev Neurosci 2014;15:300-12.

16 Sierra A, Abiega O, Shahraz A, Neumann H. Janus-faced microglia: beneficial and detrimental consequences of microglial phagocytosis. Front Cell Neurosci 2013;7:6.

17 Paolicelli RC, Bolasco G, Pagani F, Maggi L, Scianni M, Panzanelli P, et al. Synaptic pruning by microglia is necessary for normal brain development. Science 2011;333:1456-8.

18 Schafer DP, Lehrman EK, Kautzman AG, Koyama R, Mardinly AR, Yamasaki R, et al. Microglia sculpt postnatal neural circuits in an activity and complement-dependent manner. Neuron 2012;74:691-705.

19 Filipello F, Morini R, Corradini I, Zerbi V, Canzi A, Michalski B, et al. The microglial innate immune receptor TREM2 is required for synapse elimination and normal brain connectivity. Immunity 2018;48:979-991.e8.

20 Stevens B, Allen NJ, Vazquez LE, Howell GR, Christopherson KS, Nouri N, et al. The classical complement cascade mediates CNS synapse elimination. Cell 2007;131:116478.

21 Hong S, Beja-Glasser VF, Nfonoyim BM, Frouin A, Li S, Ramakrishnan S, et al. Complement and microglia mediate early synapse loss in Alzheimer mouse models. Science 2016;352:712-6.

22 Rajendran L, Paolicelli RC. Microglia-Mediated Synapse Loss in Alzheimer's Disease. J Neurosci 2018;38:2911-9.

23 Sellgren CM, Gracias J, Watmuff B, Biag JD, Thanos JM, Whittredge PB, et al. Increased synapse elimination by microglia in schizophrenia patient-derived models of synaptic pruning. Nat Neurosci 2019;22:374-85.

24 Team RC. R: A language and environment for statistical computing. R Foundation for Statistical Computing, Vienna, Austria. 2012. URL http://www R

25 Boche D, Perry VH, Nicoll JAR. Review: activation patterns of microglia and their identification in the human brain. Neuropathol Appl Neurobiol 2013;39:3-18.

26 Franco-Bocanegra DK, McAuley C, Nicoll JAR, Boche D. Molecular mechanisms of microglial motility: changes in ageing and alzheimer's disease. Cells 2019;8. doi:10.3390/cells8060639

27 Minett T, Classey J, Matthews FE, Fahrenhold M, Taga M, Brayne C, et al. Microglial immunophenotype in dementia with Alzheimer's pathology. J Neuroinflammation 2016;13:135.

28 Shen F, Jiang L, Han F, Degos V, Chen S, Su H. Increased Inflammatory Response in Old Mice is Associated with More Severe Neuronal Injury at the Acute Stage of Ischemic Stroke. Aging Dis 2019;10:12-22.

29 Arnold SE, Trojanowski JQ, Gur RE, Blackwell P, Han LY, Choi C. Absence of neurodegeneration and neural injury in the cerebral cortex in a sample of elderly patients with schizophrenia. Arch Gen Psychiatry 1998;55:225-32.

30 De Picker LJ, Morrens M, Chance SA, Boche D. Microglia and Brain Plasticity in Acute Psychosis and Schizophrenia Illness Course: A Meta-Review. Front Psychiatry 
2017;8:238.

31 Koffie RM, Meyer-Luehmann M, Hashimoto T, Adams KW, Mielke ML, Garcia-Alloza $\mathrm{M}$, et al. Oligomeric amyloid beta associates with postsynaptic densities and correlates with excitatory synapse loss near senile plaques. Proc Natl Acad Sci USA 2009;106:4012-7.

32 Spires-Jones TL, Hyman BT. The intersection of amyloid beta and tau at synapses in Alzheimer's disease. Neuron 2014;82:756-71.

33 DeKosky ST, Scheff SW. Synapse loss in frontal cortex biopsies in Alzheimer's disease: correlation with cognitive severity. Ann Neurol 1990;27:457-64.

34 Terry RD, Masliah E, Salmon DP, Butters N, DeTeresa R, Hill R, et al. Physical basis of cognitive alterations in Alzheimer's disease: synapse loss is the major correlate of cognitive impairment. Ann Neurol 1991;30:572-80.

35 Carpanini SM, Torvell M, Morgan BP. Therapeutic inhibition of the complement system in diseases of the central nervous system. Front Immunol 2019;10:362.

36 Sekar A, Bialas AR, de Rivera H, Davis A, Hammond TR, Kamitaki N, et al. Schizophrenia risk from complex variation of complement component 4 . Nature 2016;530:177-83.

37 Comer AL, Jinadasa T, Sriram B, Phadke RA, Kretsge LN, Nguyen TPH, et al. Increased expression of schizophrenia-associated gene $\mathrm{C} 4$ leads to hypoconnectivity of prefrontal cortex and reduced social interaction. PLoS Biol 2020;18:e3000604.

38 Oosterhof N, Chang IJ, Karimiani EG, Kuil LE, Jensen DM, Daza R, et al. Homozygous Mutations in CSF1R Cause a Pediatric-Onset Leukoencephalopathy and Can Result in Congenital Absence of Microglia. Am J Hum Genet 2019;104:936-47.

39 Dejanovic B, Huntley MA, De Mazière A, Meilandt WJ, Wu T, Srinivasan K, et al. Changes in the Synaptic Proteome in Tauopathy and Rescue of Tau-Induced Synapse Loss by C1q Antibodies. Neuron 2018;100:1322-1336.e7.

40 Shi Q, Chowdhury S, Ma R, Le KX, Hong S, Caldarone BJ, et al. Complement C3 deficiency protects against neurodegeneration in aged plaque-rich APP/PS1 mice. Sci Transl Med 2017;9. doi:10.1126/scitranslmed.aaf6295

41 Tzioras M, Daniels MJ, King D, Popovic K, Holloway RK, Stevenson AJ, et al. Altered synaptic ingestion by human microglia in Alzheimer's disease. BioRxiv Published Online First: 7 October 2019. doi:10.1101/795930

42 Li S, Cai Z, Wu X, Holden D, Pracitto R, Kapinos M, et al. Synthesis and in vivo evaluation of a novel PET radiotracer for imaging of synaptic vesicle glycoprotein $2 \mathrm{~A}$ (SV2A) in nonhuman primates. ACS Chem Neurosci 2019;10:1544-54.

43 Chen M-K, Mecca AP, Naganawa M, Finnema SJ, Toyonaga T, Lin S-F, et al. Assessing synaptic density in alzheimer disease with synaptic vesicle glycoprotein $2 \mathrm{~A}$ positron emission tomographic imaging. JAMA Neurol 2018;75:1215-24.

44 Colom-Cadena M, Spires-Jones T, Zetterberg H, Blennow K, Caggiano A, DeKosky ST, et al. The clinical promise of biomarkers of synapse damage or loss in Alzheimer's disease. Alzheimers Res Ther 2020;12:21. 
bioRxiv preprint doi: https://doi.org/10.1101/2020.05.22.110759; this version posted May 25, 2020. The copyright holder for this preprint (which

was not certified by peer review) is the author/funder, who has granted bioRxiv a license to display the preprint in perpetuity. It is made available under aCC-BY 4.0 International license.

474 Supplementary Table 1: Linear mixed-effects model outcomes from R Studio.

475

476

\begin{tabular}{|c|c|c|c|}
\hline Measurement & Effect size & $\begin{array}{c}\text { Standard } \\
\text { Error }\end{array}$ & p-value \\
\hline $\begin{array}{c}\text { Iba1 burden } \\
\text { (\% area) }\end{array}$ & -0.1637 & 0.1580 & 0.315 \\
\hline $\begin{array}{l}\text { CD68 burden } \\
(\% \text { area })\end{array}$ & 0.0165 & 0.06215 & 0.794 \\
\hline $\begin{array}{l}\text { CD68+Iba1 } \\
\text { colocalisation } \\
(\% \text { area })\end{array}$ & -0.0378 & 0.0792 & 0.639 \\
\hline $\begin{array}{l}\text { CD68+Syn1 } \\
\text { colocalisation } \\
\text { (\% area })\end{array}$ & -0.0772 & 0.0917 & 0.413 \\
\hline $\begin{array}{l}\text { CD68+Syn1 } \\
\text { colocalisation } \\
\text { /CD68 (\% area) }\end{array}$ & -0.1414 & 0.0980 & 0.167 \\
\hline $\begin{array}{l}\text { CD68+Syn1 } \\
\text { colocalisation } \\
\text { /Iba1 (\% area) }\end{array}$ & -0.0044 & 0.0958 & 0.964 \\
\hline
\end{tabular}

